A Comparison of IRT Theta Estimates and Delta Scores

From the Perspective of Additive Conjoint Measurement

\author{
Benjamin W. Domingue ${ }^{1}$ \\ \& \\ Dimiter M. Dimitrov ${ }^{2}$
}

${ }^{1}$ Stanford University

2 George Mason University. 


\begin{abstract}
A recently developed framework of measurement, referred to as Delta-scoring (or $D$-scoring) method (DSM; e.g., Dimitrov 2016, 2018, 2020) is gaining attention in the field of educational measurement and widely used in large-scale assessments at the National Center for Assessment in Saudi Arabia. The $D$-scores obtained under the DSM range from 0 to 1 to indicate how much (what proportion) of the ability measured by a test of binary items is demonstrated by the examinee. This study examines whether the $D$-scale is an interval scale and how $D$-scores compare to IRT ability scores (thetas) in terms of intervalness via testing the axioms of additive conjoint measurement (ACM). The approach to testing is the ConjointChecks (Domingue, 2014), which implements a Bayesian method to evaluating whether the axioms are violated in a given empirical item response data set. The results indicate that the $D$-scores, computed under the DSM, produce fewer violations of the ordering axioms of ACM than do the IRT "theta" scores. The conclusion is that the DSM produces a dependable $D$-scale in terms of the essential property of intervalness.
\end{abstract}




\section{A Comparison of IRT Theta Estimates and Delta Scores From the Perspective of Additive Conjoint Measurement}

\section{Introduction}

A recently developed approach to test scoring, equating, and item analysis, which is gaining attention in the field of educational and psychological assessment, is referred to as delta scoring method (or D-scoring method, DSM; Dimitrov, 2016, 2018, 2020; Dimitrov \& Atanasov, 2021). Currently, the DSM is adopted by the Education \& Training Evaluation Commission (ETEC) in Saudi Arabia for college admission, teacher certification, and other large-scale assessments. The DSM is designed for dichotomously scored items but, as shown by Dimitrov and Luo (2018), it can be readily used with ordered polytomous items and the results parallel their counterparts obtained under the graded response model (GRM) in IRT (Samejima, 1969, 1996).

The DSM is developed in a classical framework, DSM-C (Dimitrov, 2016, 2018, 2020) and latent framework, DSM-L (Dimitrov \& Atanasov, 2021), sharing a common $D$-scale and key psychometric concepts and interpretations. The $D$-scale ranges from 0 to 1 , with $D=0$ when none of the test items is answered correctly and $D=1$ when all items are answered correctly. The $D$-score of an examinee indicates how much (what proportion) of the ability required for total success on the test is demonstrated by that examinee. The DSM-C extends the traditional classical test theory (CTT) by introducing (a) a scoring approach that takes into account the examinee's response pattern and item difficulties, (b) regression modeling of item response functions (IRFs), (c) conditional standard error of measurement, and other psychometric features. The DSM-L provides an "IRT-like" estimation of item and person parameters on the $D$-scale. It should be emphasized that the DSM can be used as a practically efficient alternative to IRT in the context of some assessments, but not to replace IRT as a comprehensive framework of measurement. Some information on this matter are provided later in this paper, but for details on the DSM and its comparison to CTT and IRT, the reader may refer to previous DSM studies (e.g., Dimitrov, 2016, 2018, 2020; Dimitrov \& Atanasov, 2021; Dimitrov \& Luo, 2019).

\section{Purpose of the Study}

A key question about the psychometric quality of the $D$-scale, not addressed in previous studies, is whether it is an interval scale and how $D$-scores compare to IRT ability scores (thetas) in terms of intervalness. With the purpose of addressing this question, this paper is organized as 
follows. First, a brief description of the DSM (classical and latent estimations) is provided in the next section. Second, methods relevant to addressing the question about the intervalness of the $D$-scale in comparison with the IRT ability scale are discussed. Of main interest is the question which score ( $D$ or theta) is more consistent with the axioms of additive conjoint measurement (ACM; Luce \& Tukey, 1964). Third, this question is addressed through the approach of ConjointChecks (Domingue, 2014) with the use of real data from a large-scale assessment at the NCA. Fourth, the trade-off between stringency and violations is examined in the full dataset. The last section contains a discussion focusing on the question of whether the $D$-scores or the IRT theta-scores are more consistent with the axioms of ACM.

\section{The $D$-Scoring Method (DSM)}

\section{Classical DSM (DSM-C)}

Under the DSM-C, the $D$-score of an examinee on a set of test items is based on his/her response vector of (1/0) item scores and the expected difficulties of the items, $\delta_{i}$. It should be clarified that $\delta_{i}=1-\pi_{i}$, where $\pi_{i}$ is the expected 'easiness' of the item; that is, the expected proportion of correct item responses for a targeted population of examinees. Specifically, once the $\delta_{i}$ values are estimated for a test of $n$ binary items (e.g., via bootstrapping), the $D$-score of person $s$ on the test is computed as follows

$$
D_{s}=\frac{\sum_{i=1}^{n} X_{s i} \delta_{i}}{\sum_{i=1}^{n} \delta_{i}}
$$

where $X_{s i}$ is the score (1/0) of person $s$ on item $i$. Clearly, $0 \leq D s \leq 1$, with $D s=0$ if the answers of all items are incorrect $\left(X_{s i}=0 ; i=1, \ldots, n\right)$ and $D s=1$ if all answers are correct; that is, $X_{s i}=1 ; i=1, \ldots, n$. The $D$-score can be interpreted as the proportion (\%) of the ability needed for a total success on the test demonstrated by the examinee. For ease of interpretation in test reports, the $D$-scores can be rescaled to range from 0 to 100 .

Item Response Function (IRF) on the D-Scale. A key feature of the DSM is the twoparameter rational function model (RFM2) which is used to obtain IRFs on the $D$-scale (Dimitrov, 2020). The analytic form of RFM2 is

$$
P=\frac{1}{1+\left[\frac{b(1-D)}{D(1-b)}\right]^{S}},
$$


where $P$ is the probability of correct item response, $D$ is the person's test score on the $D$-scale (from 0 to 1 ), $b$ is the item location on the $D$-scale (that is, the location where the probability of correct item response is 0.5 ). and $s$ is a fit parameter for shape. The item discrimination, a, is obtained "post-hoc" as a function of the parameters $b$ and $s$ as follows (see Dimitrov, 2019)

$$
a=\frac{s}{4 b(1-b)} .
$$

The RFM2 model can be reduced to a one-parameter model (RFM1) by fixing $s=1$ or extended to a three-parameter model (RFM3) by introducing a pseudo-guessing parameter, $c$, as it is done in IRT (e.g., Hambleton, Swaminathan, \& Rogers, 1991).

Under the classical DSM-C, Equation 2 is treated as a nonlinear regression where the person's score, $D$ (computed in advance via Equation 1), is used as a predictor of the person's item score ( 1 or 0 ) and the item parameters $b$ and $s$ are estimated as regression coefficients. Under the latent DSM-L, Equation 2 is treated as a latent model where the $D$-scores are not known in advance but, instead, estimated along with the item parameters using a maximumlikelihood procedure (Dimitrov \& Atanasov, 2021). The RFM2 model can be reduced to a oneparameter model (RFM1) by fixing $\mathrm{s}=1$ in Equation 2 or extended to a three-parameter model (RFM3) by introducing a pseudo-guessing parameter, $c$, as it is done in IRT (e.g., Hambleton, Swaminathan, \& Rogers, 1991).

\section{Latent DSM (DSM-L)}

Under the DSM-L, the regression model in Equation 2 is treated as a latent model for simultaneous estimation of the item parameters ( $b$ and $s$ ) and person parameter (latent $D$-score) using maximum likelihood estimator (MLE) or a different approach such as Markov Chain Monte Carlo (MCMC) method as it is done in IRT, but on the D-scale [0-1] (e.g., see Sheng, 2008, for MCMC estimations in IRT).

\section{Brief Notes on DSM-C and DSM-L}

The DSM-C and DSM-L share the same $D$-scale and same analytic forms of IRFs but differ in their approaches to estimation of item and person parameters. Previous studies showed that the estimates of $D$-scores under the DSM-L are in general more accurate than their DSM-C counterpart, but their values are highly correlated ( $r \approx 0.99$; e.g., Dimitrov \& Atanasov, 2021). In both cases, the conditional standard error of $D$-scores decreases toward the ends of the $D$-scale 
(i.e., 0 and 1), which is just the opposite of the case in IRT where the standard error increases towards the ends of the selected range on the logit scale (e.g., -4 and +4 ). Thus, compared to IRT, the DSM yields higher accuracy of test scores for examinees with lower or higher levels of the ability measured by the test (e.g., students at risk or highly performing students). Provided in Appendix 1 are examples of IRFs and conditional standard errors of $D$-scores on the $D$-scale.

In line with the purpose of this study, the next sections deal with comparing $D$-scores to IRT ability scores (thetas) in terms of intervalness. Under the method of testing of axioms of additive conjoint measurement, presented next, the results are obtained for $D$-scores computed under the DSM-C for a large sample of real data. The level of intervalness of such $D$-scores can be seen as a benchmark for the intervalness of $D$-scores obtained under the DSM-L given that the DSM-L yields even more accurate estimates of $D$-scores, compared to DSM-C, and the $D$-scores under either approach are highly correlated. Thus, $D$-scores obtained via Equation 1 (under the DSM-C) are used in this study. To facilitate computations, the denominator in Equation 1 (that is, $\left.\sum_{i=1}^{n} \delta_{i}\right)$ is omitted because it is a constant and, therefore, does not influence the intervalness of the resulting $D$-scale.

\section{Method}

\section{Testing the Axioms of Additive Conjoint Measurement}

Of particular interest is which score is more consistent with the axioms of additive conjoint measurement (ACM; Luce \& Tukey, 1964). Evaluating these axioms in fallible data is not easy. The approach used here, ConjointChecks (Domingue, 2014), builds off earlier work (Karabatsos, 2001) in implementing a Bayesian method of evaluating whether the axioms are violated in a given empirical item response data set. That approach is naturally applied to data scaled via the Rasch (1993) model in which the sum score is a sufficient statistic for student ability. IRT theta and $D$-score estimates of student ability are not discrete and this raises questions about how to apply the ConjointChecks approach to violation detection. This issue is resolved by introducing a metric meant to determine the stringency of a banding (defined as the discretization of the non-discrete theta or $D$-score estimates). Trade-offs between the banding stringency and the axiom violations associated with a given banding can then be examined.

Ability estimates of $\theta_{s}$, which are called here "theta" scores for simplicity, are frequently used as estimates of student ability in practice. The estimates used here are maximum likelihood 
estimates obtained via Xcalibre (Guyer \& Thompson, 2013). However, there are potential problems associated with the 3PL if the goal is "measurement" in a rigorous sense (e.g., Wright, 1997). Both the $D$ and theta scores have one noteworthy trait: they are not discrete. Rasch (1993) ability estimates are discrete in the sense that they take only values a finite set of values (that set being in a 1-1 mapping with the sum scores). As show in the following, this is problematic from the perspective of the ConjointChecks approach.

ACM (Luce \& Tukey, 1964) is an axiomatic approach to defining a measure when direct concatenation is not possible. A crucial point is that evaluation of whether data is consistent with the axioms of ACM is not a question of whether a data is consistent with a given model (e.g., the $3 \mathrm{pl}$ ). Rather, it is a question of whether the data supports a certain interpretation. In particular, it is known that an interval scale (in this case, for student ability) exists when the axioms of additive conjoint measurement hold within a given dataset. The axioms are primarily concerned with order. In the case of item response data, orderings amongst the probabilities for individuals (at different abilities) responding to items (at different difficulties) are of interest. The ConjointChecks approach (Domingue, 2014) is an implementation of an algorithm for checking the axioms of ACM given the fact that item responses contain error. In particular, it uses an approach originally suggested by Karabatsos (2001) where the necessary orderings are enforced upon the data via the jumping distribution of a Metropolis-Hastings algorithm. The question is then whether the observed proportion of correct responses for a given set of respondents assumed to be at some common ability to some item is consistent with the posterior distribution of the probabilities for correct responses generated by the Metropolos-Hastings algorithm. If not, then the ConjointChecks algorithm is said to have detected a "violation". The violation percentages $(\mathrm{Vp})$ are checked in 3x3 sub-matrices of the full data matrix. These matrices are either formed via a random selection of items and groups of individuals or via the collection of adjacent items and groups of individuals.

The ConjointChecks approach is readily applied to discrete ability estimates due to the above emphasized point that requires identification of a group of respondents thought to be at a common ability. The fact that $D$ and theta scores are continuous makes this challenging since the only guaranteed common ability from these approaches occur when students have the same item response patterns. Since there are $2^{N}$ item response patterns for $N$ items, this may not be a frequent occurrence. To get around this dilemma, we consider different "bandings" of the D and 
theta scores. A banding is defined as a division of the real number line. Essentially, it is a discretization of the continuous number line. No banding is perfect (e.g., some individuals in a given band may be very near a cutpoint defining a band while others are precisely between two cutpoints), but it is clear that some bandings are more "stringent" than others in the sense that they are more likely to place a person in the wrong band given the error known to be associated with either a person's D or theta estimate.

We define stringency in the following way. Given a banding, each estimate falls into a certain band of the banding. We then use the SE associated with that estimate and the normal approximation to compute the probability that the estimate is actually within the band. For individual $i$, denote this probability as $p_{i}$. Stringency is then defined as

$$
-\sum_{i} \log p_{i}
$$

For an individual, a more stringent banding will result in a smaller $p_{i}$. The logarithmic transformation will then generate a negative number with magnitude increasing as $p_{i}$ decreases. We take the negative of the sum so that larger values on this metric indicate more stringent bandings. Figure 1 offers a simple example of stringency. In particular, the banding is defined via the cutpoints at $-1.5,0$, and 1 (the dashed vertical lines). There are two individuals with ability estimates of -2 and 0.25 (note that the abilities are estimates with different amounts of precision (indicated by the width of the normal approximation). For each individual, the proportion of the normal approximation within the band of the banding that their estimate falls (the gray areas) are the $p_{i}$. These are 0.75 and 0.37 for the individuals with estimates -2 and 0.25 respectively. Thus the stringency is

$$
-(\log 0.75+\log 0.37)=1.3
$$

Intuition behind stringency Vp-trade off. If the stringency of the banding was increased via the addition of a cutpoint at -3 , this would reduce 0.75 to 0.66 . The stringency would then rise to 1.4.

The reason for the introduction of the stringency measure is as follows. Recall that a key requirement before implementing the ConjointChecks algorithm is that we need a grouping of individuals thought to be at a common level of ability. Intuitively, we might expect that a more stringent banding would produce fewer axiom violations. The plan will be to examine the violation percentages produced by common bandings of the $D$ and theta scores as compared to their respective stringencies. 


\section{Real Data and NCR Results}

Analyses in this study are based on responses of 45,612 Saudi teacher candidates to 79 items. The data come from a Teacher Certification Test (TCT) developed and administered by the National Center for Assessment in Riyadh, Saudi Arabia. The examinees are teachers who apply for certification. The correlation between theta and $D$-scores is 0.91 . Theta and $D$-score are correlated at 0.96 and 0.98 respectively with the sum scores. Figure 2 compares the theta and $D$ scores as well as their standard errors ( $S E$ s). Figure 2A shows that the relationship between the theta and $D$-scores is not perfectly linear despite the high correlation. Figure 2B focuses on the SEs. There is a dramatic difference between them. This is an important fact since these SEs play a role in the definition of banding stringency. Figure $2 \mathrm{C}$ demonstrates the classic $\mathrm{U}$-shaped relationship between theta estimate and the SEs of theta. In contrast, Figure 2D does not demonstrate anywhere near as clear a pattern. The reason why is shown in Figures $2 \mathrm{E}$ and $2 \mathrm{~F}$. Figure 2E shows that theta $S E$ s do not vary substantially in magnitude. That is not the case for the magnitude of D SEs, shown in Figure 2F.

In a set of preliminary analyses, violation percentages based on the "natural" banding of the sum scores were computed for a set of 5000 respondents. For the continuous abilities, we considered mean score (either theta or $D$ ) for individuals at a given sum score. The banding is then defined by the midpoints between all consecutive means. I consider Vp from two types of checks. The first looks at all adjacent 3-matrices from the full data matrix while the second considers 5,000 randomly chosen 3-matrices. I also consider unweighted Vp and a weighted Vp where violations at a given portion of the scale are weighted based on the number of individuals at that part of the scale. Additional details can be found in Domingue (2014). In simulations, the weighted Vp indicator in the randomly chosen 3-matrices seemed to be optimal so we focus on that metric here. Results are shown in Table 1. The sum scores generate the smallest percentages of violations (for all $\mathrm{Vp}$ ), but this is perhaps to be expected given that this banding is based on the sum scores. The $D$-scores look very similar to the sum scores, especially on the weighted metrics. The theta scores produce more violations, notably more so for the randomly chosen 5,000 3-matrices. Note that the banding seems to be more stringent for the theta score. However, given the results in Figure 2B, it might be foolhardy to compare stringency metrics across theta and $D$ scales. There is no obvious banding available for the continuous theta and $D$-scores, so we now turn to the stringency/Vp tradeoff for a number of potential bandings. 


\section{Stringency/Vp Tradeoff}

To investigate the effect that banding stringency had on $\mathrm{Vp}$,we considered a number of bandings. Bandings are characterized by two points. The first characteristic is the number of cutpoints. Cutpoints are always evenly spaced. We varied the number between 10 and 190 in increments of $20(10,30, \ldots)$. Given that there are 79 items, there are 80 bands in the sum score banding which is roughly the middle of the range used here. The second characteristic is the starting point of the first band in the banding. For example, the banding of Figure 1 would be slightly different if the first cutpoint was moved to -1 from -1.5 . The first cutpoint is either at the $0.005,0.01$, or 0.015 quantile of the score distribution. Intervals are then evenly spaced across the scale of the abilities with the last cutpoint being at either the $0.985,0.99$, or 0.995 quantile of the score distribution depending upon the choice of the first cutpoint (the span of the intervals always being $99 \%$ of the distribution). For both scales, the banding is defined within the scale (unlike in Section 3, when we used the sum score banding as the basis for bandings for theta and $D$ ) so that the $\mathrm{Vp}$ computed based on a choice of banding are optimal for each scale.

Figure 3 shows the 4 types of Vp (weighted/unweighted; adjacent versus randomly formed 3-matrices) versus banding stringency. In each figure, the $\mathrm{x}$-axis is the stringency while the $y$-axis shows the $\mathrm{Vp}$. Figures 3C and 3D have the most important results. Focus first on the black numbers which are the theta estimates. The numbers indicate the number of cutpoints in the banding. One can see that for a choice of this number (e.g., 10), there is not much difference in either the stringency of the banding or the Vp computed based on the banding. More importantly, as the stringency of the scale increases, we can observe a clear decline in the Vp. Thus the intuition with respect to banding stringency in Section 2 does seem to hold true. The same pattern is clear with the $D$-score estimates, in red. More importantly, the $D$-scores consistently produce lower $\mathrm{Vp}$ both for a fixed number of cutpoints within a banding as well as overall (the Vp based on the most stringent banding for theta is very similar to the Vp based on the least stringent banding for $D$ ). This can be clearly observed in Table 2 . For example, the $D$ scores produce a mean weighted $\mathrm{Vp}$ of 0.075 for bandings with 10 cutpoints while this is also the weighted Vp produced by theta scores with 190 cutpoints.

\section{Conclusion}

This study provides consistent evidence that the $D$-scores, computed under the classical DSM method (DSM-C; Dimitrov, 2016, 2018, 2020) produce fewer violations of the ordering axioms 
of ACM than do the theta scores. This is seen for several approaches for assigning individuals to a group of common ability. The first such approach is via the sum scores. Under this approach, the $D$-scores produce violations at rates comparable to those of the sum scores themselves, which is interesting since the grouping based on sum scores is presumably optimal for the sum scores. The more extensive investigation in Section 4 shows that the $D$-scores produce fewer violations for bandings based upon a large range of cutpoints.

The level of intervalness of $D$-scores obtained under the DSM-C is expected to be even higher for $D$-scores obtained under the latent DSM (DSM-L; Dimitrov \& Atanasov, 2021). This is because (a) the DSM-L produces even more accurate estimates of $D$-scores, and (b) $D$-scores obtained under the DSM-C and DSM-L are very highly correlated (typically, $r \approx 0.99$ ) (e.g., see Dimitrov \& Atanasov, 2021). Thus, under either approach to estimation, the DSM produces a dependable $D$-scale in terms of the essential psychometric property of intervalness. 


\section{References}

Dimitrov, D. M. (2020). Modeling of item response functions under the $D$-scoring method. Educational and Psychological Measurement, 80(1), 126-144 (online first in 2019, DOI: 10.1177/0013164419854176).

Dimitrov, D. M. (2018). The delta scoring method of tests with binary items: A note on true score estimation and equating. Educational and Psychological Measurement, 78(5), 805-825.

Dimitrov, D. M. (2016). An approach to scoring and equating tests with binary items:

Piloting with large-scale assessments. Educational and Psychological Measurement, 76, 954-975.

Dimitrov \& Atanasov (2021). Latent $D$-scoring modeling: Estimation of item and person parameters. Educational and Psychological Measurement, 81(2) 388-404 [published online first in 2020, DOI: 10.1177/0013164420941147].

Dimitrov, D. M., \& Luo, Y. (2019). A Note on the $D$-scoring method adapted for polytomous test items. Educational and Psychological Measurement, 79(3), 545-557 (online first in 2018, DOI: 10.1177/0013164418786014).

Domingue, B. (2014). Evaluating the equal-interval hypothesis with test score scales. Psychometrika, 79, 1-19.

Guyer, R., \& Thompson, N.A. (2013). User's Manual for Xcalibre item response theory calibration software, version 4.2. St. Paul MN: Assessment Systems Corporation.

Hambleton, R. K., Swaminathan, H., \& Rogers, H. (1991). Fundamentals of item response theory. Newbury Park, CA: Sage.

Karabatsos, G. (2001). The Rasch model, additive conjoint measurement, and new models of probabilistic measurement theory. Journal of Applied Measurement, 2, 389-423.

Luce, R. D., \& Tukey, J. W. (1964). Simultaneous conjoint measurement: A new type of fundamental measurement. Journal of Mathematical Psychology, 1, 1-27.

Rasch, G. (1960). Probabilistic models for intelligence and attainment tests. Copenhagen: Danmarks Paedagogiske Institut.

Sheng, Y. (2008). Markov chain Monte Carlo estimation of normal ogive IRT models in MATLAB. Journal of Statistical Software, 25(8), 1-15. https://doi.org/10.18637/ jss.v025.i08

Wright, B. D. (1997). A history of social science measurement. Educational Measurement: Issues and Practice, 16, 33-45. 


\section{Appendix 1}

\section{Examples of Item Characteristic Curves and Conditional Standard Error of $D$-scores}

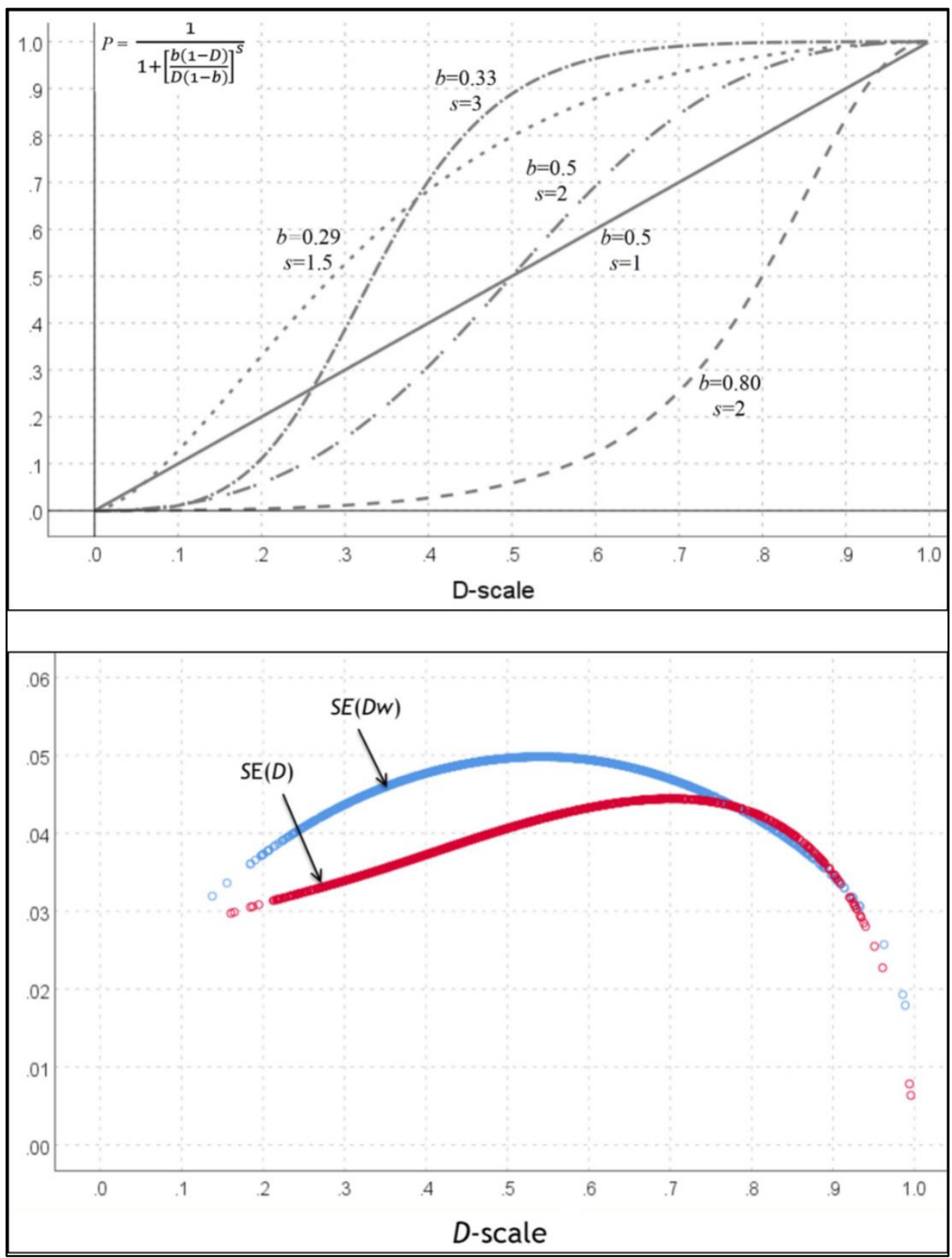

Note. The item characteristic curves (upper panel) are for five items with different item location, b, and shape parameter, s, under the RFM2. In the lower panel, $S E\left(D_{\mathrm{w}}\right)$ is the standard error of the "weighted" $D$-scores, $D_{\mathrm{w}}$, computed via Equation 2 under the DSM-C, whereas $S E(D)$ is the standard error estimated under the DSM-L (see Dimitrov \& Atanasov, 2020). 


\section{Appendix 2}

\section{Frequency distributions of the errors associated with $D$-scores and IRT theta-scores}

\begin{tabular}{|c|c|c|c|}
\hline Frequency & Stem & $\&$ & Distribution of $D$-score errors \\
\hline 2907.00 & 0 & . & 0000000000111111112222222223333333334444444445555555556666666666777777777888888888899999999 \\
\hline 2816.00 & 1 & . & 00000000011111111122222222233333333444444444455555555566666666777777778888888899999999 \\
\hline 2796.00 & 2 & . & 000000000111111111222222222333333333444444445555555556666666667777777788888888999999999 \\
\hline 2783.00 & 3 & . & 00000000001111111122222222333333333444444444555555555666666666777777778888888899999999 \\
\hline 2722.00 & 4 & . & 0000000001111111122222222233333333344444444555555556666666677777777788888888999999999 \\
\hline 2542.00 & 5 & . & 0000000011111111122222223333333344444445555555566666666777777777888888889999999 \\
\hline 2399.00 & 6 & . & 0000000011111111222222223333333344444445555555566666666777777788888889999999 \\
\hline 2351.00 & 7 & . & 0000000011111112222222233333334444444555555566666667777777788888889999999 \\
\hline 2207.00 & 8 & . & 000000011111122222223333333444444455555556666666777777788888889999999 \\
\hline 2079.00 & 9 & . & 0000000111111122222233333334444455555556666667777777888888999999 \\
\hline 1969.00 & 10 & . & 00000011111112222223333334444445555555666666777777888888999999 \\
\hline 1869.00 & 11 & . & 0000001111122222333333444444555556666667777778888889999999 \\
\hline 1691.00 & 12 & . & 0000011111122222333334444455555566666777778888899999 \\
\hline 1573.00 & 13 & . & 0000001111222223333344444555556666677777888889999 \\
\hline 1465.00 & 14 & . & 0000001111122222333344445555666667777888889999 \\
\hline 1306.00 & 15 & . & 0000111122223333444455556666777788889999 \\
\hline 1257.00 & 16 & . & 000011112222333344445555666677778888999 \\
\hline 1123.00 & 17 & . & 00001111222333444555566667778888999 \\
\hline 990.00 & 18 & . & 0001112222333444555666777888999 \\
\hline 795.00 & 19 & . & 001112223334455666778899 \\
\hline 795.00 & 20 & . & 0001122233344556677788899 \\
\hline 683.00 & 21 & . & 00112233445566778899 \\
\hline 622.00 & 22 & . & 00112233445566778899 \\
\hline 530.00 & 23 & . & 001122334455677899 \\
\hline 453.00 & 24 & . & 01223345667789 \\
\hline 424.00 & 25 & . & 012233456789 \\
\hline 344.00 & 26 & . & 0123456789 \\
\hline 328.00 & 27 & . & 0123456789 \\
\hline 283.00 & 28 & . & 0123456789 \\
\hline 221.00 & 29 & . & $012345678 \&$ \\
\hline 201.00 & 30 & . & 012345688 \\
\hline 96.00 & 31 & . & 01348 \\
\hline 992.00 & Extremes & & $(>=3.15)$ \\
\hline
\end{tabular}

\begin{tabular}{|c|c|c|c|}
\hline Frequency & Stem & & Distribution of theta-score errors \\
\hline 4.00 & 29 & . & $\&$ \\
\hline 79.00 & 30 & . & $\& \&$ \\
\hline 353.00 & 31 & . & $456789 \& \&$ \\
\hline 791.00 & 32 & . & 0123445566778899 \\
\hline 1233.00 & 33 & . & 001112223344455666777888999 \\
\hline 1798.00 & 34 & . & 00011112223333444555566667777888889999 \\
\hline 2531.00 & 35 & . & 00001111122222333334444445555556666667777777888889999999 \\
\hline 3541.00 & 36 & . & 000000011111112222222333333344444445555555666666666777777778888888899999999 \\
\hline 4186.00 & 37 & . & 000000001111111122222222333333333344444444555555555666666666677777777788888888899999999999 \\
\hline 4350.00 & 38 & . & 000000001111111111222222222333333333344444444455555555556666666667777777778888888888999999999 \\
\hline 4111.00 & 39 & . & 000000000111111111222222222333333333444444444555555555666666666777777778888888888999999999 \\
\hline 3652.00 & 40 & . & 0000000011111111222222223333333344444445555555566666666777777788888889999999 \\
\hline 3044.00 & 41 & . & 00000001111111222222233333334444445555556666666777777888888999999 \\
\hline 2398.00 & 42 & . & 000000111112222233333444445555566666777778888899999 \\
\hline 2061.00 & 43 & . & 00000111112222333334444555566667777788889999 \\
\hline 1701.00 & 44 & . & 000011122223333444455556667778888999 \\
\hline 1428.00 & 45 & . & 0001112222333444555666777888999 \\
\hline 1197.00 & 46 & . & 0001112223334455666778899 \\
\hline 929.00 & 47 & . & 00112233445566778899 \\
\hline 862.00 & 48 & . & 0011223344556778899 \\
\hline 693.00 & 49 & . & 001122334456789 \\
\hline 562.00 & 50 & . & 01233456789 \\
\hline 516.00 & 51 & . & 0123456789 \\
\hline 392.00 & 52 & . & 0123456789 \\
\hline 376.00 & 53 & . & 0123456789 \\
\hline 64.00 & 54 & . & 01 \\
\hline 2760.00 & Extremes & & $(>=.542)$ \\
\hline
\end{tabular}




\section{Table 1}

Violation Percentages (Vp) for Natural Banding of Sum Scores (NCR)

\begin{tabular}{rccccc}
\hline & \multicolumn{2}{c}{ Vp-Adj } & \multicolumn{2}{c}{ Vp-5k } & Stringency \\
\hline & Unwt & Wt & Unwt & Wt & \\
NCR & 0.37 & 0.34 & 0.09 & 0.05 & \\
Theta & 0.41 & 0.37 & 0.14 & 0.07 & 10857 \\
D & 0.38 & 0.34 & 0.1 & 0.05 & 7735 \\
\hline
\end{tabular}




\section{Table 2}

Vp as a Function of Stringency for Checks of 5,000 Randomly Chosen 3-Matrices

\begin{tabular}{ccccccc}
\hline \multirow{2}{*}{$\begin{array}{c}\text { Number of } \\
\text { cutpoints }\end{array}$} & Stringency & Vp_W & Vp_U & Stringency & Vp_W & Vp_U \\
\hline 10 & 34304 & 0.111 & 0.183 & 23642 & 0.075 & 0.114 \\
30 & 80848 & 0.095 & 0.151 & 57403 & 0.059 & 0.089 \\
50 & 103852 & 0.088 & 0.139 & 76889 & 0.057 & 0.084 \\
70 & 119041 & 0.085 & 0.133 & 90537 & 0.056 & 0.081 \\
90 & 130380 & 0.081 & 0.128 & 100008 & 0.055 & 0.078 \\
110 & 139424 & 0.083 & 0.131 & 109658 & 0.052 & 0.076 \\
130 & 146916 & 0.081 & 0.129 & 116413 & 0.052 & 0.076 \\
150 & 153385 & 0.079 & 0.128 & 121540 & 0.052 & 0.075 \\
170 & 158947 & 0.077 & 0.127 & 125229 & 0.053 & 0.077 \\
190 & 164008 & 0.075 & 0.126 & 132421 & 0.050 & 0.075 \\
\hline
\end{tabular}

Note. Means are taken over results based on same number of cutpoints that define a banding. 


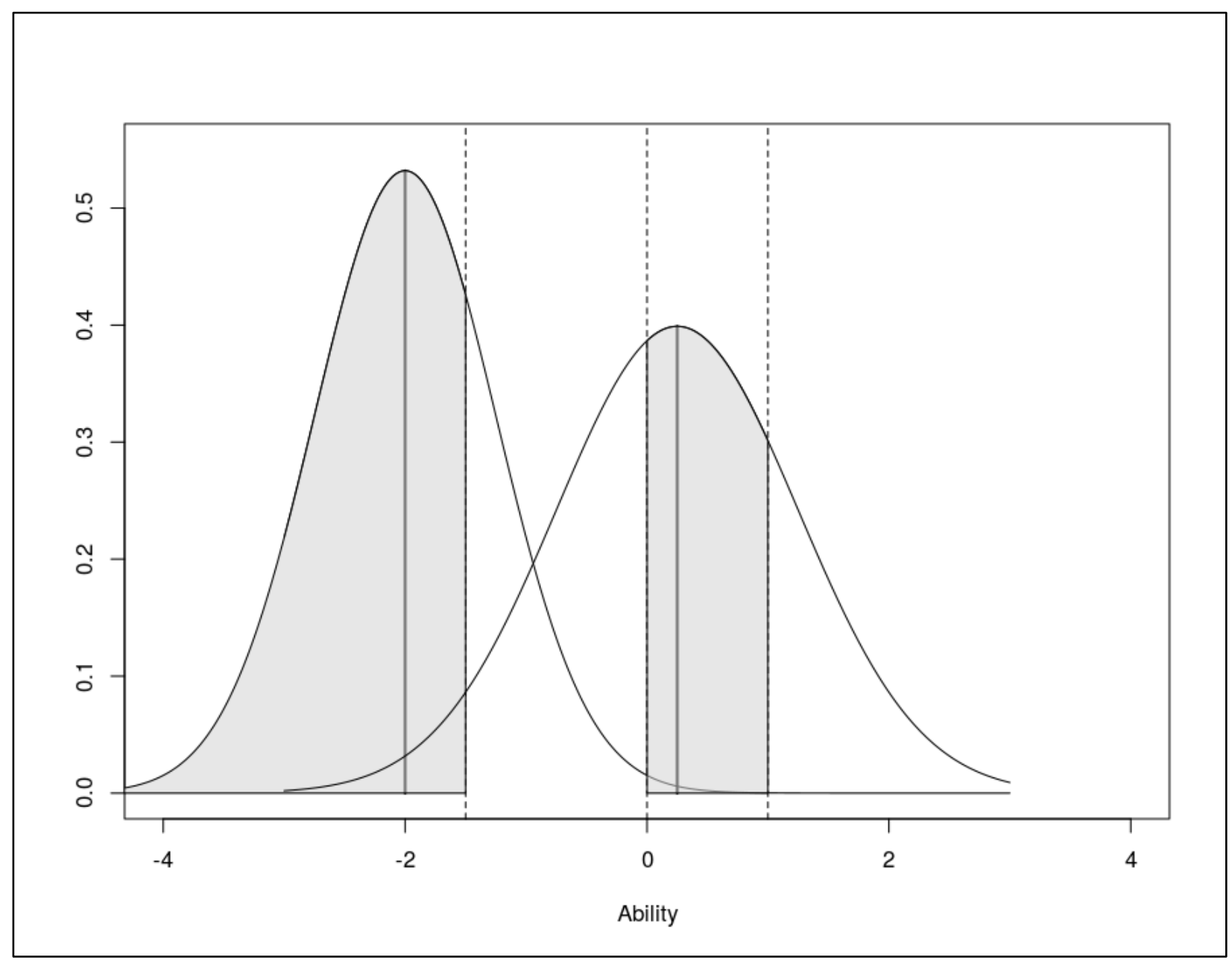

Figure 1. A simple example of stringent bindings. 

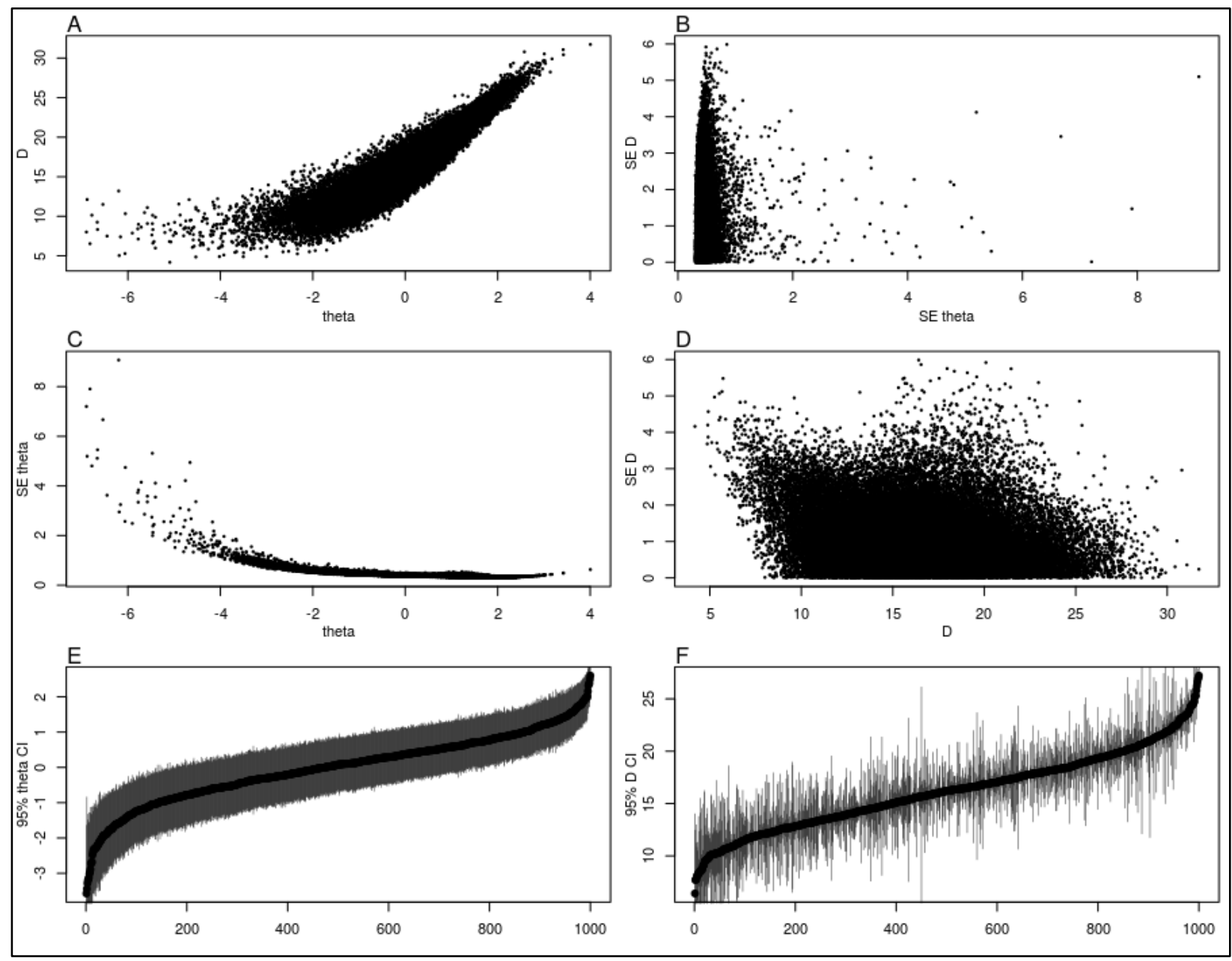

Figure 2. Comparisons of ability estimates and standard errors. 


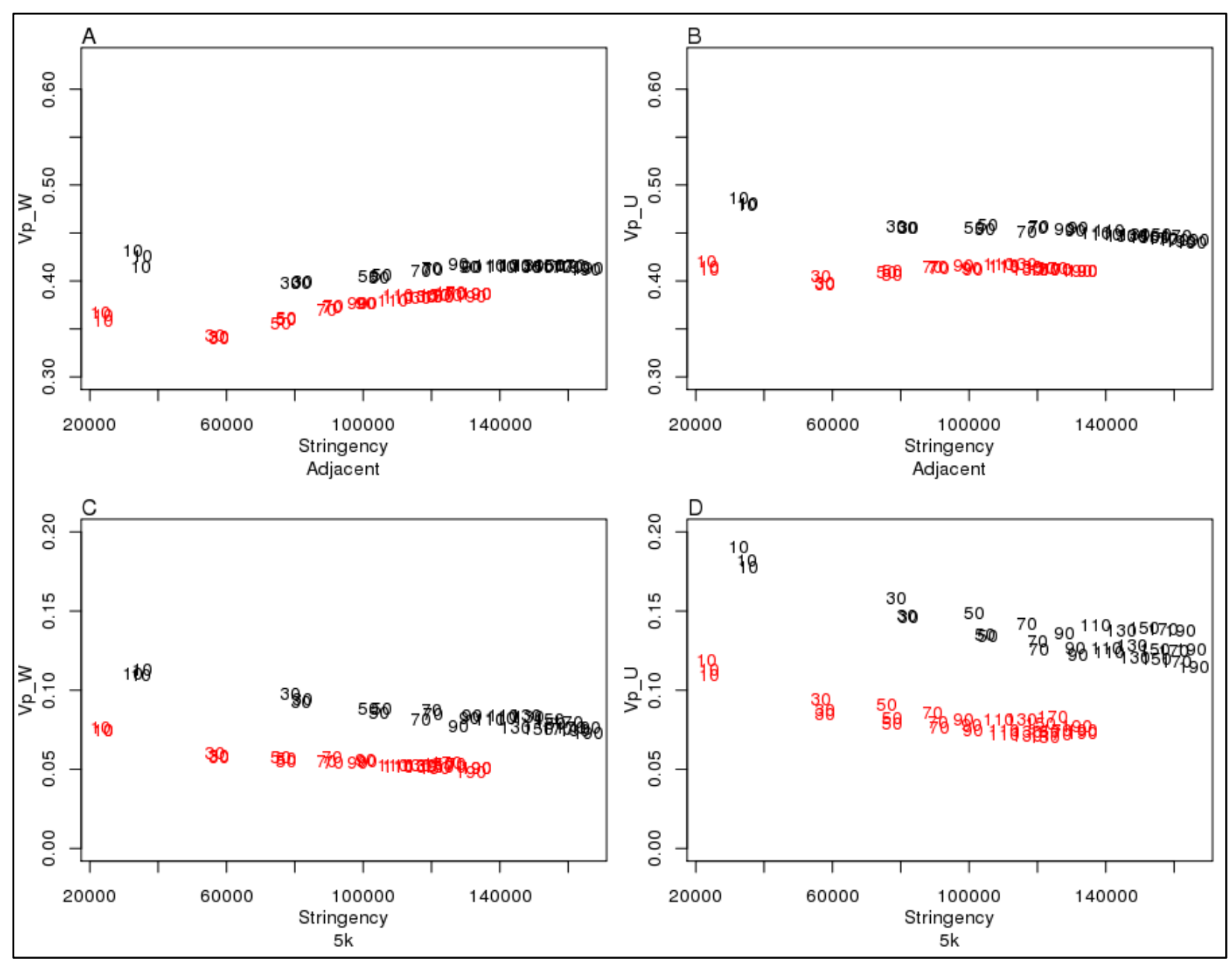

Figure 3. Tradeoff between Vp and stringency. 